\title{
Konkurrenz um Menschenrechte in Europa: die EU und der Europarat
}

\author{
Klaus Brummer*
}

Gibt es in Europa, dem Kontinent mit dem weltweit engmaschigsten Netz von sich mit Menschenrechtsfragen befassenden Institutionen, ,zuviel` Menschenrechtsschutz? Welche Ebene im europäischen Mehrebenensystem schützt die Menschenrechte am besten: die nationale oder die europäische? Die ,Solange-Rechtsprechung ' des deutschen Bundesverfassungsgerichts illustriert das Problem. ${ }^{1}$ Und wie können auf den einzelnen Ebenen die Akteure, die sich dem Menschenrechtsschutz verschrieben haben, zusammenarbeiten, ohne dass Ressourcen verschwendet werden oder Schutzlücken und widersprüchliche Standards entstehen? Diese Frage gilt insbesondere für die europäische Ebene, wo es ein nicht-hierarchisches Nebeneinander von Akteuren sowie Schutz- und Kontrollmechanismen gibt.

Aus der gleichzeitigen Existenz verschiedener den Menschenrechtsschutz in Europa verfolgender Akteure kann viel Positives folgen. Gelangen die Akteure zu einem komplementären Vorgehen, bei dem die jeweiligen Stärken gefördert, Doppelarbeiten vermieden und stattdessen Synergien geschaffen werden, kann das nur der Überwachung und Durchsetzung der Menschenrechte in Europa dienen. Die Kehrseite besteht darin, dass die Befassung mit ein und derselben Thematik leicht zu Konkurrenzsituationen zwischen den Akteuren führen kann, etwa um politischen Einfluss, finanzielle Ressourcen oder mediale Präsenz. Laut Neukirch ist Konkurrenzdenken sogar charakteristisch für den Umgang von Organisationen untereinander. ${ }^{2}$ Institutionelle Eigeninteressen können dazu führen, dass nicht nur in allgemeiner Hinsicht Potenziale verschenkt und Ressourcen verschwendet werden, die sich aus einer strategisch konzipierten und gezielt implementierten Zusammenarbeit mit anderen Organisationen ergäben. Konkret für den Menschenrechtsschutz kann die fehlende Kopplung von Organisationen zu widersprüchlichen Schutzstandards oder zu Doppelungen auf der einen und Überwachungslücken auf der anderen Seite führen.

In diesem Beitrag soll die Problematik anhand des Zusammenspiels von Europarat und Europäischer Union im Bereich des Menschenrechtsschutzes untersucht werden. Es gilt, die institutionellen und konzeptionellen Grundlagen der Zusammenarbeit der beiden Organisationen auf ihre Funktionsfähigkeit und Praktikabilität hin zu untersuchen. Zunächst werden die Fundamente der Kooperation anhand der Vereinbarungen dargestellt, die zwischen Europarat und Europäischer Union geschlossen wurden. Es zeigt sich, dass die Menschenrechtsthematik erst relativ spät berücksichtigt wurde, was eine Zusammenarbeit in der Praxis jedoch nicht ausgeschlossen hat. Anschließend richtet sich der Blick auf zwei aktuelle Probleme beziehungsweise Streitpunkte zwischen Europarat und Europäischer Union im Bereich des Menschenrechtsschutzes: die Einrichtung einer EU-Grundrechteagentur sowie die Untersuchung

* Dr. Klaus Brummer, Wissenschaftlicher Assistent, Institut für Politische Wissenschaft, Friedrich-AlexanderUniversität Erlangen-Nürnberg.

1 Siehe Heinrich Pehle: Wer schützt die Grundrechte in der Europäischen Union? Grundsätzliche Überlegungen anlässlich der Nichtigkeitserklärung des Europäischen Haftbefehlgesetzes durch das Bundesverfassungsgericht, in: Gesellschaft, Wirtschaft, Politik 1/2006, S. 25-36; zudem das Interview mit dem Präsidenten des Bundesverfassungsgerichts Hans-Jürgen Papier: „Das muss sich ändern“, in: Frankfurter Allgemeine Zeitung, 24.07.2007, S. 5.

2 Vgl. Claus Neukirch: Konfliktmanagement und Konfliktprävention im Rahmen von OSZE-Langzeitmissionen. Eine Analyse der Missionen in Moldau und Estland, Baden-Baden 2003, S. 86. 
der sogenannten ,CIA-Affäre'. Die Beispiele legen den Schluss nahe, dass die vielbeschworene Zusammenarbeit auf den Grundsätzen von Komplementarität und der Schaffung von Synergien eher auf dem Papier als in der Praxis existiert. Im nächsten Schritt werden die jüngsten Versuche geschildert, die Kooperation beider Organisationen auf eine neue Grundlage zu stellen, die den vereinbarten Prinzipien zur praktischen Durchsetzung verhelfen soll. Während in einzelnen Fragen Fortschritte zu beobachten sind, bleiben Anspruch und Wirklichkeit des Zusammenspiels von Europarat und Europäischer Union allerdings bereits auf der Ebene der schriftlichen Absprachen weiterhin defizitär. Der Beitrag endet mit einer Bewertung der jüngsten Reformbemühungen und deren Folgen für den Menschenrechtsschutz in Europa.

\section{Der Anspruch: komplementäre Partner}

Die Beziehungen zwischen Europäischer Union und Europarat wurden nach und nach ausgeweitet und vertieft. Während lange Zeit institutionelle Aspekte beziehungsweise Verfahrensfragen im Mittelpunkt standen, rückten in den letzten Jahren zunehmend Sachfragen, einschließlich des Menschenrechtsschutzes, in den Mittelpunkt der zwischen den beiden Organisationen geschlossenen Vereinbarungen. Die Anfänge der Beziehungen zwischen der heutigen Europäischen Union und dem Europarat liegen mehrere Jahrzehnte zurück. Bereits in Artikel 230 des ,Vertrags zur Gründung der Europäischen Wirtschaftsgemeinschaft ‘ vom März 1957 findet sich der, freilich wenig konkrete, Hinweis, dass ,[d]ie Gemeinschaft jede zweckdienliche Zusammenarbeit mit dem Europarat" in die Wege leiten soll. ${ }^{3}$

Ein Briefwechsel vom August 1959 zwischen dem Generalsekretär des Europarats, dem Präsidenten der Kommission der Europäischen Wirtschaftsgemeinschaft (EWG) und dem Präsidenten der Europäischen Atomgemeinschaft (Euratom) regelte anschließend grundlegende Fragen des Austausches zwischen den drei Organisationen. Es ging vor allem um die wechselseitige Unterrichtung der Beteiligten über ihre jeweiligen Aktivitäten. Wegen der Konzentration auf Verfahrensfragen, aber auch, weil zum damaligen Zeitpunkt Fragen des Menschenrechtsschutzes weder im Kontext der Europäischen Wirtschaftsgemeinschaft noch bei Euratom - im Gegensatz zum Europarat - eine Rolle spielten, enthielt der Briefwechsel keine weiteren Hinweise zu dieser Thematik.

Vielfältige, zum Teil parallel ablaufende Veränderungen und Umbrüche in den 1980er und 1990er Jahren (zum Beispiel Ende des Ost-West-Konflikts, Gründung der Europäischen Union, Erweiterung des Europarats) machten eine Anpassung der Zusammenarbeit zwischen dem Europarat und der Europäischen Gemeinschaft beziehungsweise später der Europäischen Union notwendig. Der Briefwechsel von 1959 wurde zunächst durch einen Briefwechsel vom Juli 1987 ersetzt. Dessen Inhalte wurden anschließend im November 1996 durch einen weiteren Briefwechsel bestätigt und aktualisiert. ${ }^{4}$ Erneut standen Verfahrensfragen im Mittelpunkt. Weiterhin ging es in erster Linie darum, wie der Austausch zwischen den beiden Organisationen gewährleistet werden kann.

Eine Entwicklung ergab sich insofern, als neben der wechselseitigen Unterrichtung über Aktivitäten nun auch die Einbeziehung von Vertretern der Partnerorganisation bei der Ausarbeitung von Inhalten konkretisiert wurde. So hielt der Briefwechsel von 1987 beispiels-

3 Dieser Artikel ist Art. 303 EGV (Nizza). Im Primärrecht von EU/EG (Stand: Nizza) finden sich weitere Bezüge zum Europarat. Thematische Verweise beziehen sich auf Fragen der Bildung (Art. 149 Abs. 3 EGV) und Kultur (Art. 151 Abs. 3 EGV). Zudem führt Art. 6 Abs. 2 EUV an, dass die EU die in der Europäischen Menschenrechtskonvention festgeschriebenen Grundrechte achtet. Diese Verweise auf den Europarat unterscheiden sich von der Satzung der Straßburger Organisation, in der sich keine Bezüge zur EU finden.

4 Die Briefwechsel sind abrufbar unter: http://ec.europa.eu/external_relations/coe/arrange.pdf (letzter Zugriff: 15.08.2007). 
weise fest, dass Kommissionsvertreter zu Treffen des Ministerkomitees des Europarats eingeladen werden können, um sich über den Fortschritt des europäischen Integrationsprozesses und über weitere Fragen von gemeinsamem Interesse auszutauschen. Zudem sollten Treffen der Arbeitsgruppen des Ministerkomitees des Europarats oder Fachministerkonferenzen der Organisation für die Europäische Gemeinschaft, repräsentiert durch die Kommission, geöffnet werden. Vertreter des Sekretariats des Europarats wiederum sollten ihrerseits als Beobachter in die Expertenausschüsse der Kommission eingeladen werden.

Die Menschenrechtsthematik als solche hingegen wurde in beiden Briefwechseln nicht thematisiert. Eher war das Gegenteil der Fall: Bei der Anregung, einen regelmäßigen Austausch zwischen dem Sekretariat des Europarats und der Kommission zu Fragen von wechselseitigem Interesse zu etablieren, wurden im Briefwechsel von 1987 zwar einzelne Themenbereiche explizit erwähnt. Zu diesen gehörten Rechtsfragen, Soziales, Gesundheit, Bildung und Kultur. Der Menschenrechtsschutz als solcher wurde nicht angeführt.

Die Nichtberücksichtigung der Thematik in den Briefwechseln hieß jedoch nicht, dass Menschenrechtsfragen bei der Kooperation der beiden Organisationen in der Praxis keine Rolle gespielt hätten. Zentral waren und sind noch heute die ,Gemeinsamen Programme'. Diese ko-finanzierten, zumeist länderspezifischen Maßnahmen werden seit 1993 durchgeführt und versuchen, den Zielländern - all jene Staaten, die seit 1989 dem Europarat beigetreten sind - bei rechtlichen und institutionellen Reformen, inklusive Menschenrechtsfragen, zu helfen. Die Türkei wird beispielsweise bei der Umsetzung von Menschenrechtsreformen unterstützt, die Ukraine und die Staaten des Südkaukasus bei der Förderung einer ,Kultur der Menschenrechte " und Russland bei der Stärkung von Menschenrechten und Rechtsstaatlichkeit. ${ }^{5}$

Außerdem gab es von den Briefwechseln losgelöste institutionelle Entwicklungen. Von besonderer Bedeutung war die Einführung von Spitzengesprächen zwischen Europarat und Europäischer Union in Form von ,Quadripartite-Treffen' ${ }^{6}$ Seit deren Einführung im Jahr 1989 kommen ein- bis zweimal pro Jahr unter anderem der Vorsitzende des Ministerkomitees und der Generalsekretär des Europarats mit Vertretern der EU-Ratspräsidentschaft und der Kommission zusammen. Bei diesen Treffen wird regelmäßig auch die Zusammenarbeit der beiden Organisationen in Menschenrechtsfragen diskutiert. ${ }^{7}$

In einer Vereinbarung zusammengebracht wurden die institutionellen Erwägungen und der Umgang mit Menschenrechtsfragen im April 2001. Der damalige Generalsekretär des Europarats, Walter Schwimmer, und der damalige Außenkommissar als Vertreter der Europäischen Gemeinschaft, Chris Patten, unterzeichneten eine ,Joint Declaration on Co-operation and Partnership between the Council of Europe and the European Commission ${ }^{6}{ }^{8}$ Im

5 Eine Übersicht über die ,Gemeinsamen Programme“ ist abrufbar unter: http://www.jp.coe.int/CEAD/JP/ (letzter Zugriff: 15.08.2007).

6 Angestoßen wurden diese durch Committee of Ministers: On the Future Role of the Council of Europe in European Construction, Resolution (89) 40, 05.05.1989, Art. 5.

7 Zuletzt ging es im Februar 2007 beispielsweise um die thematische wie geografische Ausrichtung der EUGrundrechteagentur und den Beitritt der EU zur EMRK. Vgl. Committee of Ministers: 24th Quadripartite Meeting between the Council of Europe and the European Union. Common Pressline of the Two Presidencies. Brussels, 13 February 2007.

8 Die Vereinbarung geht auf die Empfehlung eines, Committee of Wise Persons` zurück. Der Ausschuss, dessen Einrichtung vom zweiten Gipfeltreffen des Europarats im Jahr 1997 angestoßen wurde, sollte Vorschläge zur strukturellen Reform des Europarats vorlegen, die infolge der Erweiterung der Organisation für notwendig erachtet wurde. In dem im November 1998 vorgelegten Abschlussbericht des Ausschusses finden sich unter Punkt I.3 („A Europe of interlocking institutions“) Vorschläge zur Intensivierung der Zusammenarbeit zwischen Europarat und EU. Hierzu gehörte der Abschluss eines Rahmenabkommens zwischen den beiden Organisationen. Vgl. Committee of Ministers: Committee of Wise Persons. Final Report to the Committee of Ministers, CM (98) 178, 20.10.1998. 
Gegensatz zur, wie beschrieben, eher strukturellen Ausrichtung der Briefwechsel ging es in der Erklärung vor allem um thematische Fragen, genauer um gemeinsame Zielsetzungen der beiden Organisationen sowie um Wege, diese Ziele zu erreichen. ${ }^{9}$ Ebenfalls im Unterschied zu den Briefwechseln wurde der Menschenrechtsschutz prominent angeführt. Neben Demokratie und Rechtsstaatlichkeit wird der Schutz von Menschenrechten als gemeinsame Wertebasis wie auch als gemeinsames Ziel der beiden Organisationen benannt. Zugleich finden sich verschiedene Verpflichtungen beider Seiten, ihre geteilten Ziele auch gemeinsam zu verfolgen, nicht zuletzt durch die schon erwähnten ,Gemeinsamen Programme'. Dabei sollen ganz gezielt Länder und Themen identifiziert werden, bei denen gemeinsames Handeln einen Mehrwert erbringt.

\section{Die Realität: ein ,wackeliges Tandem“}

Die für die Kooperation zwischen Europarat und Europäischer Union zentralen Prinzipien (vor allem Komplementarität, Vermeidung von Doppelarbeit) ${ }^{10}$ und Themen (unter anderem Menschenrechtsschutz) wurden somit in der ,Gemeinsamen Erklärung ‘ von 2001 festgelegt. In verschiedenen Bereichen werden diese Grundsätze auch gelebt. Exemplarisch hierfür stehen die geschilderten ,Gemeinsamen Programme', deren Entwicklung, Finanzierung und Implementierung beide Organisationen gemeinsam tragen. Auch die Beziehungen zwischen dem Europäischen Gerichtshof für Menschenrechte (EGMR) und dem Europäischen Gerichtshof entwickeln sich positiv. ${ }^{11}$

Gleichwohl kommt der luxemburgische Premierminister Jean-Claude Juncker in seinem im April 2006 vorgelegten und weiter unten noch ausführlicher diskutierten Bericht über den gegenwärtigen Zustand der Beziehungen zwischen Europarat und Europäischer Union zu einem eher ernüchternden Fazit. Mit Blick auf die grundsätzlichen Potenziale konstatiert Juncker zwar, dass sich die beiden Organisationen ,,auf das Engste“ ergänzen würden. ${ }^{12}$ Die Bewertung der Umsetzung dieser Potenziale fällt hingegen wenig schmeichelhaft aus: „Wenngleich die beiden [Europarat und Europäische Union; KB] sich natürlich wechselseitig bereichert haben, bilden sie doch selbst heute noch bestenfalls ein wackeliges Tandem. Wenngleich sie vieles von dem jeweils anderen entlehnt haben, ist es ihnen doch zu keinem Zeitpunkt möglich gewesen, sich auf eine dauerhaft komplementäre Art und Weise aufzustellen." ${ }^{13}$ Als Beleg für diese Bewertung dienen die folgenden, seit der Erklärung von 2001 neu aufgetretenen Reibungen und Spannungen zwischen Europarat und Europäischer Union. Die Beispiele zeigen, dass die propagierten Grundsätze nicht durchweg charakteristisch für die Interaktion der beiden Organisationen sind. Das betrifft sowohl grundsätzliche institutionelle Aspekte, wie die Diskussion um die EU-Grundrechteagentur zeigt, als auch die Behandlung spezifischer inhaltlicher Fragen wie der ,CIA-Affäre‘.

9 Die Deklaration ist abrufbar unter: http://www.jp.coe.int/programmes/general/JointDeclaration_EF.asp (letzter Zugriff: 18.07.2007).

10 Bauers Untersuchung der Kulturpolitik ist ein weiteres Beispiel dafür, dass Doppelarbeiten zwischen Europarat und EU als Resultat von verbesserungswürdigen Kooperationsmechanismen und Kompetenzüberschneidungen auftreten. Vgl. Hans-Joachim Bauer: Der Europarat nach der Zeitenwende 1989-1999. Zur Rolle Straßburgs im gesamteuropäischen Integrationsprozess, Hamburg 2001, S. 260-263.

11 Laut Bergmann existiert eine ,ernsthafte und umfassende Kooperation“ zwischen den beiden Gerichtshöfen, zumal der EuGH bestrebt sei, ,,in Sachen Grundrechtsschutz kein Quäntchen Raum zwischen sich und Straßburg [dem EGMR; KB] zu lassen.“ Vgl. Jan Bergmann: Das Bundesverfassungsgericht in Europa, in: Europäische Grundrechte-Zeitschrift 20-21/2004, S. 620-627, hier S. 623-624.

12 Jean-Claude Juncker: Europarat - Europäische Union: Eine einheitliche Zielstellung für den europäischen Kontinent, 11.04.2006, S. 2-3.

13 Juncker: Europarat - Europäische Union, 2006, S. 2. 


\section{EU-Grundrechteagentur}

Angestoßen wurde die ,Agentur der Europäischen Union für Grundrechte ' (nachfolgend: Agentur) im Dezember 2003 durch einen Beschluss des Europäischen Rates. Die Staats- und Regierungschefs einigten sich, das Mandat der in Wien bereits bestehenden ,Europäischen Stelle zur Beobachtung von Rassismus und Fremdenfeindlichkeit ' derart auszuweiten, dass sie zu einer Agentur für den Menschenrechtsschutz werden würde. Den Hintergrund für diesen Schritt bildete die im Dezember 2000 auf dem Europäischen Rat in Nizza proklamierte Grundrechtecharta der Europäischen Union, die durch den Verfassungsvertrag in das Primärrecht der Union eingehen und deren Einhaltung von der Agentur überprüft werden sollte. ${ }^{14}$ Auf dieser Grundlage trieb die Kommission die Vorarbeiten an der Agentur voran, insbesondere durch eine Mitteilung im Oktober 2004 und darauf aufbauenden detaillierten Vorschlägen im Juni 2005. ${ }^{15}$ Die abschließende Einigung auf der politischen Ebene unter den Mitgliedstaaten wurde Anfang Dezember 2006 im Rat der Innen- und Justizminister erzielt. ${ }^{16}$ Am 1. März 2007 nahm die Agentur ihre Arbeit auf.

Der Europarat stand diesen Entwicklungen lange Zeit skeptisch bis ablehnend gegenüber. Die Befürchtung war, dass die Agentur inhaltlich wie geografisch in den Kernbereich der Organisation vordringen würde. Ersteres bezog sich auf den Menschenrechtsschutz in Europa, letzteres auf die Einbeziehung auch der Beitrittskandidaten und der im Rahmen der Europäischen Nachbarschaftspolitik mit der Union kooperierenden Staaten in das Aufgabenfeld der Agentur. Der Generalsekretär des Europarats, der Brite Terry Davis, kommentierte im Februar 2005 die konkreter werdenden Pläne der Europäischen Union mit den Worten: ,[W]ith all the best will in the world, I can't understand what it [die Agentur; KB] is going to do. " ${ }^{17}$ Etwas weniger deutlich, in der Sache jedoch identisch, äußerte sich im März 2005 die Parlamentarische Versammlung des Europarats. Zwar wurde der Agentur eine positive Wirkung zugesprochen, jedoch unter der Voraussetzung, dass ,,a useful role and field of action is defined for it [für die Agentur; KB] and that the agency therefore genuinely ,fills a gap" and presents irrefutable added value and complementarity in terms of promoting respect for human rights. "18 In einer im April 2006 an das Ministerkomitee adressierten Empfehlung kamen die Parlamentarier zudem auf die Gefahr von Doppelungen zwischen den Arbeiten von Institutionen des Europarats und der Agentur und deren negative Folgen für den Menschenrechtsschutz in Europa zu sprechen: „Duplication could lead to inconsistencies and create the possibility of ,forum shopping', with the countries that were subject to the different mechanisms giving preference to whichever took the more favourable position." 19

14 Der, Vertrag von Lissabon“ (Art. 6 Abs. 1 EUV) sieht nun vor, der Charta Rechtsverbindlichkeit zu verleihen.

15 Vgl. Europäische Kommission: The Fundamental Rights Agency Public consultation document. Communication from the Commission, COM (2004) 693 final, 25.10.2004; Europäische Kommission: Proposal for a Council Regulation establishing a European Union Agency for Fundamental Rights and Proposal for a Council Decision empowering the European Union Agency for Fundamental Rights to pursue its activities in areas referred to in Title VI of the Treaty on European Union, COM (2005) 280 final, 30.06.2005.

16 Die Verzögerung ergab sich durch weiter unten noch beschriebene Unstimmigkeiten zwischen den Mitgliedstaaten über das Mandat der Agentur.

17 Zitiert in Lucia Kubosova: EU discouraged from further overlap in human rights monitoring, in: EUobserver.com, 07.02.2005.

18 Parliamentary Assembly: Plans to set up a fundamental rights agency of the European Union, Resolution 1427, 18.03.2005, Art. 10.

19 Parliamentary Assembly: Follow-up to the 3rd Summit: the Council of Europe and the proposed fundamental rights agency of the European Union, Recommendation 1744, 13.04.2006, Art. 4. 
Die letztlich verabschiedeten Bestimmungen zu Aufgaben und Zielen der Agentur entsprechen den Anliegen des Europarats in weiten Teilen. ${ }^{20}$ Das Mandat der Agentur wurde geografisch wie inhaltlich begrenzt. Geografisch können sich neben den Mitgliedstaaten der Union auch die Bewerberländer sowie die Staaten des westlichen Balkans, mit denen die Union ein Stabilisierungs- und Assoziierungsabkommen geschlossen hat, als Beobachter an der Agentur beteiligen. ${ }^{21}$ In diesem Punkt fanden die Anliegen des Europarats nur teilweise Gehör, schließlich wollte er eine noch engere Begrenzung der Agentur einzig auf die Mitgliedstaaten der Union. ${ }^{22}$ Inhaltlich wiederum soll die Agentur - ganz im Sinne des Europarats - ausschließlich innerhalb des Anwendungsbereichs des Gemeinschaftsrechts aktiv werden. Laut der die Agentur begründenden Verordnung besteht deren maßgebliche Aufgabe darin, ,den relevanten Organen, Ämtern und Agenturen der Gemeinschaft und ihren Mitgliedstaaten bei der Durchführung des Gemeinschaftsrechts in Bezug auf die Grundrechte Unterstützung zu gewähren und ihnen Fachkenntnisse bereitzustellen, um ihnen die uneingeschränkte Achtung der Grundrechte zu erleichtern, wenn sie in ihrem jeweiligen Zuständigkeitsbereich Maßnahmen einleiten oder Aktionen festlegen. “23

Das insbesondere auf der Europäischen Menschenrechtskonvention (EMRK) und dem Gerichtshof für Menschenrechte beruhende Kontrollsystem des Europarats für den individuellen Menschenrechtsschutz in Europa bleibt somit unangetastet. Das ist schon allein deshalb der Fall, weil die Agentur nicht von Einzelpersonen angerufen werden und deshalb auch nicht über Einzelfälle (etwa analog zu den Individualbeschwerden vor dem EGMR) entscheiden kann. Stattdessen steht sie den Mitgliedstaaten sowie den Institutionen der Union beratend und unterstützend zur Seite, indem sie Expertise zu allgemeinen Fragen zur Verfügung stellt. Die Agentur soll insbesondere Informationen und Daten erheben, auswerten und publizieren. Dabei soll sie die Ergebnisse anderer mit Menschenrechtsfragen befasster Akteure einbeziehen. Der Europarat wird in diesem Zusammenhang ausdrücklich erwähnt. ${ }^{24}$

Diese Nennung des Europarats ist einer von mehreren Verweisen auf die Straßburger Organisation in der Agenturverordnung. Als Maxime des Zusammenspiels beider Institutionen gilt, „,Doppelarbeit zu vermeiden und Komplementarität und einen Mehrwert sicherzustellen. “25 Hierfür ist eine enge Koordination zwischen den beiden Organisationen vorgesehen. ${ }^{26}$ Dies soll gerade durch die Einbindung einer vom Europarat zu bestimmenden unabhängigen Persönlichkeit in die Institutionen der Agentur geschehen. Laut Generalsekretär Davis soll die Person „darauf aufmerksam [machen], wenn die Agentur ihr Mandat überschreitet.“27

20 Toggenburg spricht von zwei „Formen der Verdoppelungsreduktion“ - generell möglichst wenig inhaltliche Überschneidungen sowie Mechanismen zur Zusammenarbeit in gemeinsamen Bereichen -, die in der Verordnung zur EU-Agentur berücksichtigt worden seien. Vgl. Gabriel Nikolaij Toggenburg: Die EU-Grundrechteagentur: Satellit oder Leitstern? Daseinsberechtigung, Aufgaben und Herausforderungen der neuen Agentur der Europäischen Union, SWP-Aktuell 8, Februar 2007, S. 3.

21 Vgl. Verordnung (EG) Nr. 168/2007 des Rates vom 15. Februar 2007 zur Errichtung einer Agentur der Europäischen Union für Grundrechte, in: Amtsblatt der Europäischen Union, Nr. L 53 vom 22. Februar 2007, Art. 28.

22 Diese Forderung wurde von Vertretern des Europarats beispielsweise auf dem 23. Quadripartite-Treffen geäußert. Vgl. Committee of Ministers: 23rd Council of Europe/ European Union Quadripartite meeting (Brussels, 3 November 2006), CM/Inf (2006) 46 revised, 15.11.2006, Art. IIa.

23 Verordnung: Errichtung einer Agentur, 2007, Art. 2. Bezugspunkte hinsichtlich „,der Grundrechte“ sind die Bestimmungen von Art. 6 Abs. 2 EUV, und zwar, ,unter Einschluss der Europäischen Konvention zum Schutze der Menschenrechte und Grundfreiheiten“. Vgl. Verordnung, Errichtung einer Agentur, 2007, Präambel, Abs. 9.

24 Vgl. Verordnung: Errichtung einer Agentur, 2007, Art. 4 Abs. 1a.

25 Verordnung: Errichtung einer Agentur, 2007, Art. 9.

26 Laut Toggenburg enthält die Verordnung ,durchaus taugliche Bestimmungen zur Kooperation und Koordination zwischen Europarat und EU““. Toggenburg: Die EU-Grundrechteagentur, 2007, S. 4.

27 Zitiert in Frankfurter Allgemeine Zeitung: EU-Grundrechteagentur eröffnet, 02.03.2007, S. 6. 
Konkret soll die vom Europarat zu bestimmende Person Mitglied des Verwaltungsrats werden, der „Planungs- und Überwachungsinstanz“28 der Agentur, sowie an den Sitzungen des Exekutivausschusses der Agentur teilnehmen können, der den Verwaltungsrat unterstützt. Die Person besitzt allerdings nur eingeschränkte Abstimmungsrechte im Verwaltungsrat. ${ }^{29}$ Abstimmungsberechtigt ist sie etwa bei der Verabschiedung des Jahresarbeitsprogramms oder der Annahme der Jahresberichte der Agentur. Nicht abstimmen darf die vom Europarat zu bestimmende Persönlichkeit hingegen bei noch wichtigeren Führungsund Finanzfragen. Hierzu zählt die Ernennung beziehungsweise Amtsenthebung des Direktors der Agentur oder die Verabschiedung des Jahreshaushaltsplans. Dem Exekutivausschuss wiederum kann die Persönlichkeit generell nur ohne Abstimmungsrecht „,beiwohnen". ${ }^{30}$ Ein noch abzuschließendes Kooperationsabkommen soll die Zusammenarbeit zwischen Agentur und Europarat weiter spezifizieren. ${ }^{31}$

Als Folge dieser weitgehenden Berücksichtigung der Anliegen des Europarats änderte sich dessen Haltung gegenüber der Agentur. Generalsekretär Davis etwa sagte Mitte Februar 2007: „I welcome the decision by the EU Council of Ministers to create a new Fundamental Rights Agency to scrutinise EU institutions and the application of EU laws. This is an important and challenging task, and the new Agency can make a useful contribution in helping the EU to comply with the Council of Europe standards based on the European Convention on Human Rights." 32 Laut Ministerkomitee des Europarats solle das Hauptaugenmerk des Europarats nun darauf liegen, dass die Vorgaben auch in der Praxis umgesetzt würden. ${ }^{33}$

Unklar ist, zu welchem Grad dieser ,Erfolg ' des Europarats der - nicht vom Europarat als Organisation erzeugten - Kongruenz seiner Interessen mit denjenigen einzelner europäischer Staaten geschuldet ist. Nicht zuletzt angetrieben durch die Sorge um nationale Souveränitätsrechte sprachen sich Länder wie Großbritannien, Irland und die Slowakei für eine klare geografische wie vor allem inhaltliche Begrenzung der Agentur aus. ${ }^{34}$ Auch Deutschland - allen voran der EU-Ausschuss des Bundestags - setzte sich ,für eine schlanke Agentur und eine Beschränkung des geographischen Tätigkeitsbereichs auf EU und Beitrittskandidaten“35 ein. Als Gründe für die kritische Haltung des EU-Ausschusses gegenüber der Agentur nannte der damalige Ausschussvorsitzende, Matthias Wissmann, neben grundsätzlichen Problemen beim Zusammenspiel zwischen Bundestag und Bundesregierung ${ }^{36}$ die Ge-

28 Verordnung: Errichtung einer Agentur, 2007, Art. 12 Abs. 6.

29 Vgl. Verordnung: Errichtung einer Agentur, 2007, Art. 12 Abs. 8.

30 Verordnung: Errichtung einer Agentur, 2007, Art. 13 Abs. 1.

31 Vgl. Verordnung: Errichtung einer Agentur, 2007, Art. 9.

32 Council of Europe: SG - European Fundamental Rights Agency, Press Release 102a07, 15.02.2007.

33 Vgl. Committee of Ministers: Follow-up to the Third Summit: the Council of Europe and the proposed fundamental rights agency of the European Union. Parliamentary Assembly Recommendation 1744, CM/AS (2007) Rec 1744 final, 19.01.2007, Art. 5.

34 Die inhaltliche Begrenzung bezog sich vor allem darauf, ob die Agentur auch Fragen mit polizeilichem oder justiziellem Bezug untersuchen solle - und somit Themen, die nicht unter das Gemeinschaftsrecht, sondern in die Kompetenzen der Mitgliedstaaten fallen und für deren Behandlung durch die Agentur es keine Rechtsgrundlage gegeben hätte. Vgl. Lucia Kubosova: Legal dispute could delay EU rights agency, in: EUobserver.com, 12.09.2006. Allerdings verständigten sich die EU-Mitgliedstaaten darauf, bis Ende 2009 eine Überprüfung des Mandats der Agentur durchzuführen mit der Möglichkeit, dieses auf die polizeiliche und justizielle Zusammenarbeit auszuweiten. Vgl. Lucia Kubosova: EU opts for fundamental rights agency with limited scope, in: EUobserver.com, 05.12.2006.

35 Newsletter der deutschen EU-Ratspräsidentschaft: EU-Grundrechteagentur kann Tätigkeit zum 1. März 2007 aufnehmen, 15.02.2007.

36 Hier ging es um die - aus Sicht der Abgeordneten ungenügende - Berücksichtigung der Forderungen des Bundestags durch die Bundesregierung im Rahmen der europäischen Verhandlungen zur EU-Agentur. 
fahr von Kompetenzüberschreitungen der Agentur wie auch mögliche Überschneidungen mit anderen Organisationen. Wissmann hatte hier insbesondere den Europarat im Blick. ${ }^{37}$

Vor diesem Hintergrund ergeben sich mehrere Fragen. Welche Defizite gab es beim Informationsaustausch zwischen Europäischer Union und Europarat im Zuge der Konzeptionierung der Agentur, damit es zu den oben angeführten Befürchtungen und Spannungen kam? Weshalb wird der Abschluss eines Kooperationsabkommens erst nach der Gründung der Agentur - und scheinbar erst in Reaktion auf die Kritik des Europarats ${ }^{38}$ - in Angriff genommen? Die entscheidende Frage schließlich: Wenn die Interessen vor allem großer EUStaaten wie Deutschland und Großbritannien nicht denjenigen des Europarats entsprochen hätten, wäre auch dann eine auf Arbeitsteilung und die Vermeidung von Duplizierungen abzielende Regelung gefunden worden?

\section{Untersuchung der,CIA-Affäre“}

Im Europarat wurde die ,CIA-Affäre', eine Umschreibung für Geheimflüge, Entführungen und vermeintliche Geheimgefängnisse des amerikanischen Auslandsgeheimdienstes CIA in Europa, mehrgleisig verfolgt. Den Anfang machte die Parlamentarische Versammlung des Europarats im November 2005. Als Reaktion auf Medienberichte wurde der Schweizer Dick Marty, Vorsitzender des Ausschusses für Recht und Menschenrechte der Versammlung, zum Berichterstatter ernannt. Auf der Grundlage von Martys Berichten hat die Versammlung auf ihren Sitzungen im Juni 2006 und im Juni 2007 mehrere Empfehlungen und Resolutionen verabschiedet. ${ }^{39}$

Neben den eigenen Arbeiten ersuchte die Parlamentarische Versammlung Mitte Dezember 2005 die ,Europäische Kommission für Demokratie durch Recht' (,Venedig-Kommission'), eine Stellungnahme über die Rechtmäßigkeit von Geheimgefängnissen zu erstellen. In diesem Bericht sollten gerade die für die Mitgliedstaaten des Europarats aus der EMRK erwachsenden Verpflichtungen untersucht werden. Die Venedig-Kommission, ein Expertengremium, das sich vorrangig mit verfassungsrechtlichen Fragen auseinandersetzt, legte Ende März 2006 ihre Stellungnahme vor. ${ }^{40}$

Zuvor hatte bereits Ende November 2005 der Generalsekretär des Europarats, Terry Davis, auf der Grundlage von Artikel 52 EMRK $^{41}$ eine Anfrage an die Vertragsstaaten der Menschenrechtskonvention zu den rechtlichen Aspekten möglicher problematischer amerikanischer Flugbewegungen in Europa gerichtet. Davis wollte von den Staaten unter anderem wissen, ob sie angemessene Mechanismen zur Kontrolle der Aktionen von Agenten ausländischer Geheimdienste in ihrer Jurisdiktion besäßen. Eine andere Frage lautete, ob Beamte der Staaten seit Anfang 2002 wissentlich oder durch Unterlassung am Freiheitsentzug oder dem Transport von Gefangenen beteiligt waren und ob es diesbezüglich offizielle Untersu-

37 Vgl. Frankfurter Allgemeine Zeitung: Nicht noch ein Amt, 03.01.2007, S. 3.

38 Laut Kubosova haben sich die EU-Mitgliedstaaten und das Europäische Parlament erst als Reaktion auf die Kritik des Europarats auf den Abschluss eines ,Memorandum of Understanding 'verständigt. Vgl. Kubosova: Legal dispute, 12.09.2006.

39 Zuletzt angenommen wurden Parliamentary Assembly: Secret detentions and illegal transfer of detainees involving Council of Europe member states: second report, Resolution 1562, 27.06.2007; sowie Parliamentary Assembly: Secret detentions and illegal transfer of detainees involving Council of Europe member states: second report, Recommendation 1801, 27.06.2007.

40 Vgl. European Commission for Democracy Through Law: Opinion on the International Legal Obligations of Council of Europe Member States in Respect of Secret Detention Facilities And Inter-State Transport of Prisoners, Opinion no. 363/2005, 17.03.2006.

41 Art. 52 EMRK besagt: „Auf Anfrage des Generalsekretärs des Europarats erläutert jede Hohe Vertragspartei, auf welche Weise die wirksame Anwendung aller Bestimmungen dieser Konvention in ihrem innerstaatlichen Recht gewährleistet wird." 
chungen gegeben habe oder gebe. Davis veröffentlichte seinen Bericht am 1. März 2006. Mitte Juni 2006 ergänzte er seine Ausführungen durch einen Folgebericht. ${ }^{42}$

Am 15. Dezember 2005 wiederum entschied das Europäische Parlament, einen nichtständigen Ausschuss zur Untersuchung der CIA-Affäre einzusetzen. Der Beschluss fiel somit zu einem Zeitpunkt, an dem die Untersuchungen der Versammlung und des Generalsekretärs des Europarats bereits seit Wochen liefen sowie exakt an dem Tag, an welchem die Venedig-Kommission ihren Auftrag erhielt. Mitte Januar 2006 wurden Mandat und Mitglieder des 46 Personen umfassenden Ausschusses des Europäischen Parlaments bestätigt. Nach mehreren Untersuchungsreisen und Dutzenden von Anhörungen des Ausschusses legte dessen Berichterstatter, der Italiener Giovanni Claudio Fava, Ende Januar 2007 seinen Abschlussbericht vor. ${ }^{43}$ Dieser bildete die Grundlage für die Mitte Februar 2007 vom Europäischen Parlament angenommene Resolution. ${ }^{44}$ Zuvor hatte die Europäische Kommission bereits Anfang April 2006 das ,EU Network of Independent Experts on Fundamental Rights“ um eine Stellungnahme zu den CIA-Aktivitäten in Europa ersucht. Die Stellungnahme des Expertenkreises wurde Ende Mai 2006 vorgelegt und floss in die Resolution des Parlaments ein..$^{45}$

Aus dem Geschilderten ergeben sich eine inhaltliche Frage und eine Verfahrensfrage. In inhaltlicher Hinsicht ist zu diskutieren, welche neuen Ergebnisse die Untersuchung des Europäischen Parlaments erbracht hat. Dessen Abschlussresolution betont jedenfalls ausdrücklich ,die Übereinstimmung der von beiden Ausschüssen [des Europäischen Parlaments und der Parlamentarischen Versammlung des Europarats; KB] bislang erzielten Ergebnisse. ${ }^{\text {446 }}$ Die für diesen Beitrag wichtigere Frage lautet, ob die Untersuchung der CIA-Affäre - losgelöst von den Untersuchungsergebnissen als solchen - hinsichtlich der Zusammenarbeit zwischen den beiden Organisationen als Erfolg bewertet werden kann.

Zwei Punkte sind unbestreitbar. Erstens fällt die CIA-Affäre in den Aufgabenbereich der Europäischen Union. Laut Artikel 6 des EU-Vertrags von Nizza „,beruht“ die Union ,,auf den Grundsätzen der Freiheit, der Demokratie, der Achtung der Menschenrechte und Grundfreiheiten sowie der Rechtsstaatlichkeit“". Zudem findet sich in diesem Artikel die Verpflichtung der Union zur Achtung der in der Europäischen Menschenrechtskonvention festgelegten Grundrechte. Eine Untersuchung von möglichen fundamentalen Verletzungen dieser Grundrechte kann entsprechend nicht als eine Kompetenzüberschreitung gewertet werden. Zweitens hat eine Zusammenarbeit zwischen Europäischer Union und Europarat stattgefunden. So lobt beispielsweise der Berichterstatter der Parlamentarischen Versammlung des Eu-

42 Vgl. Secretary General: Secretary General's report under Article 52 ECHR on the question of secret detention and transport of detainees suspected of terrorist acts, notably by or at the instigation of foreign agencies, SG/Inf (2006) 5, 28.02.2006; sowie Secretary General: Secretary General's supplementary report under Article 52 ECHR on the question of secret detention and transport of detainees suspected of terrorist acts, notably by or at the instigation of foreign agencies, SG/Inf (2006) 13, 14.06.2006.

43 Vgl. Nichtständiger Ausschuss zur behaupteten Nutzung europäischer Staaten durch die CIA für die Beförderung und das rechtswidrige Festhalten von Gefangenen: Bericht über die behauptete Nutzung europäischer Staaten durch die CIA für die Beförderung und das rechtswidrige Festhalten von Gefangenen, A6-0020/2007, 30.01.2007.

44 Vgl. Europäisches Parlament: Entschließung des Europäischen Parlaments zu der behaupteten Nutzung europäischer Staaten durch die CIA für die Beförderung und das rechtswidrige Festhalten von Gefangenen, T60032/2007, 14.02.2007.

45 Vgl. EU Network of Independent Experts on Fundamental Rights: The Human Rights Responsibilities of the EU Member States in the Context of the C.I.A. Activities in Europe (,Extraordinary Renditions"), Opinion no. 3-2006, 25.05.2006.

46 Vgl. Europäisches Parlament: Entschließung, 14.02.2007, Art. 33. Ein konservatives Mitglied des Parlaments aus Großbritannien konstatierte, dass der EP-Untersuchungsbericht einen früheren Bericht des Europarats nur „dupliziert“ hätte. Zitiert in Financial Times.com: MEPs condemn rendition flights, 14.02.2007. 
roparats die Unterstützung, die er seitens der Europäischen Kommission erfahren hat. Diese sei gerade für den Erhalt der Informationen der Europäischen Organisation für Flugsicherung (Eurocontrol) und des EU-Satellitenzentrums über Flugbewegungen ,unschätzbar“ gewesen. ${ }^{47}$

Dennoch stellt sich die Frage, ob diese gezielte Unterstützung der laufenden Untersuchung des Europarats durch die Kommission oder durch andere EU-Institutionen nicht auch ohne parallel laufende eigenständige Aktivitäten im Rahmen der Europäischen Union möglich gewesen wäre - Aktivitäten, welche den propagierten Zielen von Kooperation und Komplementarität diametral entgegenstehen. Voraussetzung hierfür wäre eine klare Arbeitsteilung zwischen den beiden Organisationen gewesen, in diesem Fall verstanden als Federführung der einen und einer gezielten Zuarbeit der anderen. Die Federführung hätte nicht bei der Europäischen Union und deren Institutionen gelegen, sondern beim Europarat. Schließlich hatte der Europarat bereits, wie geschildert, mittels verschiedener Institutionen (Parlamentarische Versammlung, Venedig-Kommission) und Mechanismen (Artikel 52 EMRK) mit der Untersuchung der gegen die CIA erhobenen Vorwürfe begonnen. Der Union wäre die Rolle des weniger beachteten Juniorpartners zugekommen. ${ }^{48}$ In einer echten Partnerschaft wäre die inhaltliche Dimension der Aufklärung über dem Aspekt der institutionellen Verortung diesbezüglicher Aktivitäten gestanden. Dem war nicht so. Institutionelle Eigeninteressen scheinen gegenüber Effizienzerwägungen überwogen zu haben. ${ }^{49}$

\section{Auf dem Weg zu einem einheitlichen europäischen Menschenrechtsschutz?}

\section{Anstöße durch den dritten Europaratsgipfel}

Die beiden Fallbeispiele zeigen, dass die Grundlage für ein möglichst reibungsfreies und Doppelarbeiten vermeidendes Zusammenspiel zwischen Europarat und Europäischer Union noch nicht gefunden worden ist. Der jüngste Anstoß zur Fortentwicklung der Beziehungen ging vom jüngsten Gipfeltreffen des Europarats aus. Auf dem Mitte Mai 2005 unter polnischer Präsidentschaft in Warschau durchgeführten dritten Gipfel standen für deren Zukunftsfähigkeit grundlegende Themen im Mittelpunkt. Die entscheidenden internen Fragen betrafen die Sicherung der Funktionsfähigkeit des Europarats und seiner Institutionen, insbesondere des EGMR. Nach außen ging es vor allem um die langfristige Positionierung des Europarats innerhalb des europäischen Integrationsprozesses - und somit auch um die Selbstbehauptung gegenüber der Europäischen Union.

Das Gipfeltreffen mündete in der, Warschauer Erklärung ' als dem Abschlusskommuniqué des Gipfels und dem ,Aktionsplan', der die Schwerpunkte der Europaratsaktivitäten der nächsten Jahre festhält. Beide im Namen der Staats- und Regierungschefs der damals noch 46 Europaratsstaaten ${ }^{50}$ angenommenen Dokumente gingen auf das Zusammenspiel zwischen Europarat und Europäischer Union ein, und zwar in allgemeiner Hinsicht ebenso wie

47 Committee on Legal Affairs: Alleged secret detentions and unlawful inter-state transfers involving the Council of Europe member states. Draft report - Part II (Explanatory Memorandum), AS/Jur (2006) 16 Part II, 07.06.2006, S. 5.

48 Die Öffentlichkeitswirkung der eigenen Aktivitäten scheint für die Mitglieder des Ausschusses des Europäischen Parlaments eine große Rolle gespielt zu haben. Siehe Das Parlament: Fünf Fragen an: Ewa Klimt, 19./ 26.02.2007, S. 11.

49 Zugleich war das Parlament bezüglich der Bewertung der Untersuchungsergebnisse beinahe gespalten. Bei der abschließenden Abstimmung im Plenum zum Bericht des Sonderausschusses stimmten 382 Abgeordnete mit „Ja“, 256 mit „Nein“ und 74 enthielten sich. Vgl. Honor Mahoney: MEPs approve critical CIA report but remain divided, in: EUobserver.com, 14.2.2007.

50 Im Mai 2007 ist Montenegro als 47. Staat dem Europarat beigetreten. 
speziell mit Blick auf die Zusammenarbeit im Bereich des Menschenrechtsschutzes. In der Warschauer Erklärung wurde explizit die Verbindung zwischen dem Menschenrechtsschutz - laut Erklärung eines der drei „Hauptziele“ der Organisation, zu deren Durchsetzung alle Aktivitäten der Organisation beitragen sollen - und der Zusammenarbeit des Europarats mit der Europäischen Union hergestellt. ${ }^{51}$ Die Staats- und Regierungschefs des Europarats bekundeten die Absicht ,to create a new framework for enhanced co-operation and interaction between the Council of Europe and the European Union in areas of common concern". $52 \mathrm{Zu}$ diesen Feldern gemeinsamen Interesses zählte der Menschenrechtsschutz.

Die Forderung, die Zusammenarbeit mit der Europäischen Union im Bereich des Menschenrechtsschutzes auszubauen, wurde im Aktionsplan weiter spezifiziert. ${ }^{53} \mathrm{Um}$ dieses Ziel, sowie grundsätzlich eine effizientere und Synergien schaffende Kooperation der beiden Organisationen zu gewährleisten, sollten die Beziehungen durch den Abschluss eines ,Memorandum of Understanding ' auf eine neue Grundlage gestellt werden. Als Referenzpunkte für diese Absichtserklärung wurden im Anhang des Aktionsplans zehn Leitlinien angeführt. ${ }^{54}$ Unter diesen finden sich speziell bezogen auf die Zusammenarbeit beim Menschenrechtsschutz folgende drei Aspekte: Erstens solle die Europäische Union schnellstmöglich der Europäischen Menschenrechtskonvention beitreten, wodurch die Kohärenz bei Menschenrechten in Europa gesichert werden würde. Zweitens bekundete der Europarat seinen Willen, die Europäische Union auch in Zukunft im Bereich der Menschenrechte zu unterstützen und zu beraten. Drittens wurde mit Blick auf die EU-Grundrechteagentur die Hoffnung geäußert, dass diese eine Gelegenheit zur weiteren Vertiefung der Zusammenarbeit der zwei Organisationen bieten und zudem zu einer größeren Kohärenz und Komplementarität beitragen werde.

\section{Weitere Impulse durch den Juncker-Bericht}

Im Nachgang zum dritten Gipfeltreffen des Europarats wurden vier Prioritäten bei der Umsetzung der dort getroffenen Beschlüsse festgelegt. Neben der Intensivierung der Demokratieförderung und der Entwicklung eines menschlicheren Europas waren dies die Konsolidierung des Menschenrechtsschutzsystems des Europarats sowie die Pflege der Zusammenarbeit mit der OSZE und der Europäischen Union. ${ }^{55}$ Die Frage, wie die letztgenannte Kooperation verbessert werden kann, und zwar auch mit Blick auf den Menschenrechtsschutz in Europa, wurde noch auf dem Gipfeltreffen an den luxemburgischen Premierminister Jean-Claude Juncker herangetragen. Junckers Aufgabe lautete, in ,,persönlicher Eigenschaft" den Zustand der Europarat-EU-Beziehungen zu durchleuchten und Vorschläge für deren Optimierung auszuarbeiten. ${ }^{56}$

Knapp ein Jahr nach seiner Beauftragung legte Juncker im April 2006 seinen Bericht ,Europarat - Europäische Union: Eine einheitliche Zielstellung für den europäischen Kontinent‘ vor. In diesem findet sich eine Vielzahl von Einzelvorschlägen, die zu einer strategischen Partnerschaft zwischen Europarat und Europäischer Union beitragen sollen. Nachfolgend

51 Vgl. Committee of Ministers: Third Summit of Heads of State and Government of the Council of Europe (Warsaw, 16-17 May 2005). Warsaw Declaration, CM (2005) 79 final, 17.05.2005.

52 Committee of Ministers: Warsaw Declaration, 17.05.2005, Art. 10.

53 Vgl. Committee of Ministers: Third Summit of Heads of State and Government of the Council of Europe (Warsaw, 16-17 May 2005). Action Plan, CM (2005) 80 final, 17.05.2005, Kap. 4 Art. 1.

54 Vgl. Committee of Ministers: Action Plan, 17.05.2005, Appendix 1 ("Guidelines on the Relations between the Council of Europe and the European Union").

55 Vgl. Committee of Ministers: Follow-up to the Third Summit. Parliamentary Assembly Recommendation 1712, CM/AS (2006) Rec 1712 final, 20.01.2006.

56 Vgl. Committee of Ministers: Warsaw Declaration, 17.05.2005, Art. 10. 
werden die wichtigsten Anregungen hinsichtlich der Zusammenarbeit beim Menschenrechtsschutz angeführt. ${ }^{57}$ Eine der Forderungen Junckers war der umgehende Beitritt der Europäischen Union zur EMRK. Dieser Schritt würde, ,im Hinblick auf den Schutz der Menschenrechte in Europa für ein Höchstmaß an Kohärenz und Koordinierung (...) sorgen. “58

Eine zweite Forderung lautete, den Europarat als Bezugsrahmen in Menschenrechtsfragen zu etablieren. Die Europäische Union sollte nach Ansicht Junckers die Arbeiten der mit Menschenrechtsfragen befassten Institutionen des Europarats als primäre Bezugsquellen nutzen. Würden die Aktivitäten des Europarats systematisch wie auch verbindlich im Rahmen der Europäischen Union genutzt und zudem die Europaratsinstitutionen konsequent von EU-Gremien im Zuge der Entwicklung neuer Maßnahmen konsultiert, erführe die Organisation eine deutliche Aufwertung.

Drittens sprach sich Juncker für eine konsequente Nutzung des Menschenrechtskommissars des Europarats auch durch die Europäische Union aus. In Menschenrechtsfragen, welche die EU-Mitglieder berühren und die zugleich durch keine vorhandenen Kontrollmechanismen abgedeckt werden, solle es der Europäischen Union formal ermöglicht werden, den Menschenrechtskommissar einzuschalten.

Ein vierter Vorschlag bezog sich auf die Begrenzung der EU-Grundrechteagentur. Juncker verwies ,,auf die mögliche Bedrohung, welche von dieser neuen Institution für die Einheitlichkeit des Schutzes der Menschenrechte in Europa ausgehen könnte. " ${ }^{59}$ Um dieser Bedrohung zu begegnen, schlug Juncker vor, dass die Agentur komplementär zu den Überwachungseinheiten des Europarats agieren sollte. Insbesondere müsse das Mandat der Agentur auf Menschenrechtsfragen begrenzt werden, die sich aus der Umsetzung des Gemeinschaftsrechts ergäben. Eine allgemeine Überwachungsfunktion dürfe der Agentur demgegenüber nicht zukommen. Beide Punkte wurden, wie oben geschildert, bei der Gründung der Agentur berücksichtigt.

\section{Memorandum of Understanding}

Im Mai 2007 wurde auf der Grundlage der Vorgaben des Warschauer Gipfels, des Juncker-Berichts sowie weiterer Vorschläge, etwa der Parlamentarischen Versammlung des Europarats, ${ }^{60}$ ein neues Grundlagendokument zwischen Europarat und Europäischer Union unterzeichnet: das ,Memorandum of Understanding between the Council of Europe and the European Union' ${ }^{61}$ Diese Absichtserklärung setzt die Linie der ,Gemeinsamen Erklärung ‘ von 2001 in zweifacher Hinsicht fort. Zum einen wird erneut das gesamte Spektrum von thematisch-inhaltlichen bis hin zu institutionell-verfahrenstechnischen Vorgaben behandelt. Zum anderen bleiben diese Bestimmungen weiterhin wenig ambitioniert und insgesamt eher vage. ${ }^{62}$ Deshalb dürfte auch dieses Dokument nur ein weiterer Zwischenschritt auf dem Weg zu einer tatsächlich auf Arbeitsteilung und Komplementarität beruhenden Zusammenarbeit zwischen Europarat und Europäischer Union bleiben.

57 Daneben sprach sich Juncker insbesondere für eine größere Substanz der interinstitutionellen Beziehungen aus. Das gilt sowohl für die Quadripartite-Treffen als auch für die Beziehungen zwischen Parlamentarischer Versammlung und Europäischem Parlament. Zudem befürwortete Juncker einen Beitritt der EU zum Europarat bis zum Jahr 2010. Auf diese Weise könne die EU in allen Europaratsgremien für sich sprechen.

58 Juncker: Europarat - Europäische Union, 2006, S. 5.

59 Juncker: Europarat - Europäische Union, 2006, S. 9.

60 Vgl. Parliamentary Assembly: Memorandum of Understanding between the Council of Europe and the European Union, Recommendation 1743, 13.04.2006.

61 Vgl. Committee of Ministers: Memorandum of Understanding between the Council of Europe and the European Union, CM (2007) 74, 10.05.2007. 
Das Memorandum bekräftigt die anvisierten Grundprinzipien der Zusammenarbeit. Eine vertiefte Kooperation und eine verlässliche Koordination in Fragen gemeinsamen Interesses werden als Zielvorgaben angeführt. Erneut wird die Menschenrechtsthematik besonders herausgestellt, und zwar allgemein für den Europarat wie auch konkret für dessen Zusammenspiel mit der Europäischen Union. Bei letzterem wird der Schutz von Menschenrechten und Grundfreiheiten an erster Stelle bei den Feldern angeführt, in denen die Organisationen ihre Kooperation ausbauen wollen. Zu den Themenschwerpunkten gehören der Schutz von Minderheiten, der Kampf gegen Folter und Menschenhandel sowie die Förderung von Meinungs- und Informationsfreiheit. Auf der Grundlage ebenso unteilbarer wie universeller Menschenrechte gilt es aus Sicht beider Organisationen dabei, die „cohesion of the human rights protection system in Europe "63 zu bewahren. Das betrifft speziell die Kohärenz zwischen EG- und EU-Recht auf der einen und den einschlägigen Konventionen des Europarats auf der anderen Seite. Ausdrücklich erwähnt wird freilich, dass EG-/EU-Recht bereits bestehende Schutzmechanismen weiter vertiefen dürfe.

Während das Vorherige größtenteils Bekanntes wiederholt, geht das Memorandum an verschiedenen Stellen über frühere Vereinbarungen hinaus. Neu ist beispielsweise die mehrfache ausdrückliche Betonung des Europarats als Referenzpunkt für den Menschenrechtsschutz in Europa, und zwar auch für die Europäische Union. Der Europarat wird nicht nur als ,the benchmark for human rights, the rule of law and democracy in Europe"64 bezeichnet. Darüber hinaus ist angeführt, dass " $[t]$ he European Union regards the Council of Europe as the Europe-wide reference source for human rights", ${ }^{65}$ dessen Normen in den EU-Dokumenten als Referenz angeführt werden sollen. Zudem, einem der Vorschläge Junckers folgend, will die Europäische Union ihre Beziehungen zum Menschenrechtskommissar des Europarats ausbauen. Schließlich wird die Reichweite der EU-Grundrechteagentur ausdrücklich auf den Unionsrahmen begrenzt. Die Inhalte der rechtsverbindlichen Agenturverordnung werden somit im nicht bindenden Memorandum symbolisch bekräftigt.

So wenig spezifisch wie teilweise der Text selbst sind auch die zu dessen Weiterverfolgung vorgesehenen Maßnahmen. Beide Seiten verpflichten sich, die Umsetzung des Memorandums regelmäßig zu überprüfen. Auf der Grundlage der Evaluierungen soll bis spätestens 2013 ein gemeinsamer Beschluss getroffen werden, der zur Anpassung des Textes durch die Einführung neuer Schwerpunkte der Zusammenarbeit führen könnte. ${ }^{66}$

\section{Fazit}

Die Kluft zwischen Anspruch und Wirklichkeit der Zusammenarbeit von Europarat und Europäischer Union beim Menschenrechtsschutz ist offensichtlich. Bekundungen von Abstimmung und Partnerschaftlichkeit stehen in der Praxis Doppelarbeiten und Konflikte entgegen, welche das Gegenteil bezeugen. Sicher: Zusammenarbeit findet statt, und wo dies geschieht, wird auch Mehrwert erzielt. Die jüngsten Differenzen bei der Gründung der EUGrundrechteagentur und der Untersuchung der ,CIA-Affäre‘ verdeutlichen allerdings das

62 Die Unbestimmtheit beschränkt sich nicht auf die Zusammenarbeit im Menschenrechtsbereich. Vielmehr trifft sie auch auf weitere inhaltliche wie institutionelle Bereiche zu. Exemplarisch hierfür ist die Aussage, dass beide Seiten „prüfen“ werden, wie sie ihre Präsenz bei der Partnerorganisation ausbauen können - ein Hinweis, der sich vor allem auf die bislang fehlende permanente Präsenz von EU/EG beim Europarat bezieht. Vgl. Committee of Ministers: Memorandum of Understanding, 10.05.2007, Art. 51.

63 Committee of Ministers: Memorandum of Understanding, 10.05.2007, Art. 16.

64 Committee of Ministers: Memorandum of Understanding, 10.05.2007, Art. 10.

65 Committee of Ministers: Memorandum of Understanding, 10.05.2007, Art. 17.

66 Vgl. Committee of Ministers: Memorandum of Understanding, 10.05.2007, Art. 55. 
fortbestehende Koordinations- und Kooperationsproblem zwischen den beiden Organisationen beim Menschenrechtsschutz.

Beide Seiten scheinen gewillt, ihre Zusammenarbeit weiter zu verbessern. Angestoßen durch das dritte Gipfeltreffen des Europarats im Mai 2005, und bekräftigt durch den Juncker-Bericht, mündeten die Abstimmungen zwei Jahre später in ein ,Memorandum of Understanding '. In diesem wird erneut der Wille bekundet, bestehende Defizite zu überwinden und zu Komplementarität und Synergien zu gelangen. Auch diese nicht bindende Absichtserklärung stellt jedoch nur einen Zwischenschritt zur Erreichung dieser Ziele dar. Gerade die Unbestimmtheit der Inhalte lässt weiterhin viel Raum für Auslegungen, Missverständnisse und Konflikte. Eine ,Hochrangige Expertengruppe ' diskutiert gegenwärtig weitere Vorschläge des Juncker-Berichts. Ob diese umgesetzt werden, bleibt abzuwarten.

Weitergehende Impulse für die Zusammenarbeit zwischen Europäischer Union und Europarat brächte der ,Vertrag von Lissabon'. In diesem verpflichtet sich die Europäische Union zum Beitritt zur Europäischen Menschenrechtskonvention (Artikel 6 Absatz 2 EUV). ${ }^{67}$ Hierdurch würden die Organe der Union durch die Konvention gebunden, und der Europäische Gerichtshof für Menschenrechte erhielte eine unmittelbare Zuständigkeit, die Vereinbarkeit des Gemeinschaftsrechts mit der Konvention zu prüfen. ${ }^{68}$ Während der Beitritt der Union zur Menschenrechtskonvention die Kohärenz beim Menschenrechtsschutz in Europa verbessern dürfte, könnte eine andere Neuerung des Vertrags von Lissabon den gegenteiligen Effekt haben. Der Vertrag sieht vor, dass die Grundrechtecharta der Union Rechtsverbindlichkeit erhält (Artikel 6 Absatz 1 EUV). Als ein eigenständiger EU-Grundrechtekatalog eröffnet die Charta laut Alber und Widmaier dem Europäischen Gerichtshof die Möglichkeit, eine von der Menschenrechtskonvention getrennte Grundrechtsdogmatik zu entwickeln. Als Folge könnten Interpretations- und somit Rechtsprechungsdivergenzen in Zukunft wahrscheinlicher werden. ${ }^{69}$

Die entscheidenden, vornehmlich politischen Fragen blieben freilich auch nach dem Inkrafttreten des Vertrags von Lissabon unbeantwortet. Sie lauten: Wie genau darf beziehungsweise muss die Zusammenarbeit zwischen Europarat und Europäischer Union ge- und verregelt werden? Und wie viel Freiraum bedarf es? Detaillierte Festlegungen, die Schwerpunkte und Arbeitsteilungen bestimmen, würden ein Mehr an Verlässlichkeit bringen und verstärkt zu Einsparungseffekten und Synergien führen. Sie würden allerdings Reibungen oder Doppelarbeiten nicht grundsätzlich ausschließen. Politischer Wille zur Zusammenarbeit, was in manchen Fällen auch eine Zurücknahme der eigenen Organisation bedeutet, ist und bleibt entscheidend. Gerade medial prominent behandelte Themen wie die CIA-Affäre scheinen prädestiniert dafür zu sein, eine Eigendynamik zu entwickeln, bei der Absprachen zwischen Organisationen den Eigeninteressen von Organisationen nachgeordnet werden.

Politischer Wille ist es allerdings auch, der das Gegenteil bewirken kann: die praktische Umsetzung einer auf Koordination und, wo immer möglich, Kooperation beruhenden Zusammenarbeit. Von derart ineinandergreifenden Institutionen (,interlocking institutions") würden alle Beteiligten profitieren. Der Europarat könnte sein Gewicht gegenüber den

67 Ein Beitritt ist auf beiden Seiten an Voraussetzungen geknüpft. Insbesondere müsste die Union eine Rechtspersönlichkeit erhalten - was sie im Falle der Ratifizierung des Vertrags von Lissabon täte -, und die Menschenrechtskonvention, die bislang nur Staaten offen steht, müsste für den Beitritt von internationalen bzw. supranationalen Organisationen geöffnet werden - was im 14. Zusatzprotokoll zur EMKR vorgesehen ist. Das Protokoll konnte aufgrund der fehlenden Ratifizierung durch Russland jedoch noch nicht Inkrafttreten.

68 Vgl. Siegbert Alber/Ulrich Widmaier: Mögliche Konfliktbereiche und Divergenzen im europäischen Grundrechtsschutz, in: Europäische Grundrechte Zeitschrift 5-8/2006, S. 113-123, hier S. 122-123.

69 Vgl. Alber/Widmaier: Mögliche Konfliktbereiche und Divergenzen, 2006, S. 122. 
Adressatenstaaten zusätzlich erhöhen, wenn er seine Arbeiten im Bereich des Menschenrechtsschutzes in einen klar erkennbaren Bezug zur politisch wie finanziell ungleich mächtigeren Europäischen Union stellen könnte. Die Union würde ihrerseits Entlastung erfahren und sich zudem bei ihren Aktivitäten auf vom Europarat erarbeitete hochwertige Ergebnisse stützen können, ohne diese selbst erstellen oder finanzieren zu müssen. Die EU-Staaten, die allesamt auch Europaratsstaaten sind, sähen ihre Mittel effizienter eingesetzt, ohne dass die Qualität des Menschenrechtsschutzes in Europa leiden würde.

Alle von den Kontrollmechanismen von Europarat und Europäischer Union erfassten Staaten schließlich würden nicht mit möglicherweise widersprüchlichen Standards und Vorgaben beim Menschenrechtsschutz konfrontiert werden. Zugleich wäre die Gefahr des ,forum shoppings' gebannt, bei dem sich Staaten mit Defiziten beim Menschenrechtsschutz auf diejenige Organisation berufen, die ihnen weniger kritisch gegenübersteht. Durch ein abgestimmtes Vorgehen von Europarat und Europäischer Union ließe sich diese Gefahr in eine Chance für die beiden Organisationen umwandeln, ihre geteilten Ziele durch ein geeintes Vorgehen umzusetzen. Wenn die Herausforderung somit darin besteht, die Zusammenarbeit so weit zu verregeln wie nötig und zugleich so flexibel zu belassen wie möglich, sind Europarat und Europäische Union weiterhin auf der Suche nach der richtigen Balance.

\section{Forschungsstand Politikwissenschaft}

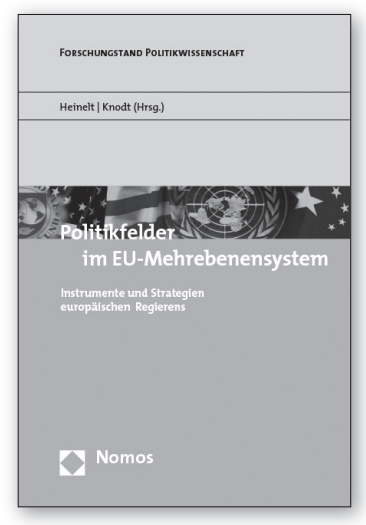

Politikfelder im EU-Mehrebenensystem Instrumente und Strategien europäischen Regierens Herausgegeben von Hubert Heinelt und Michèle Knodt 2008, ca. 350 S., brosch., ca. 29,-€, ISBN 978-3-8329-2273-3

Erscheint Juni 2008

Dieser Sammelband gibt einen Überblick über die zentralen Politikfelder der EU. Dabei wird jeweils neben der historischen Entwicklung der einzelnen Politikfelder auf die wesentlichen Steuerungsmechanismen und die maßgeblichen Akteure bei der Politikformulierung und Implementation eingegangen und nach der konkreten Ausprägung und Bedeutung einer Mehrebenenstruktur gefragt. 